\title{
Derivation of spin Hamiltonians from the exact Hamiltonian: Application to systems with two unpaired electrons per magnetic site
}

\author{
I. de P. R. Moreira, ${ }^{1,2}$ N. Suaud,${ }^{3}$ N. Guihéry, ${ }^{3}$ J. P. Malrieu, ${ }^{3}$ R. Caballol, ${ }^{4}$ J. M. Bofill,,${ }^{1,5}$ and F. Illas ${ }^{1,2}$ \\ ${ }^{1}$ Centre Especial de Recerca en Química Teòrica, Parc Cientific de Barcelona, Baldiri Reixach 4-6, E-08028 Barcelona, Spain \\ ${ }^{2}$ Department de Química Física, Universitat de Barcelona, Martí i Franquès 1, E-08028 Barcelona, Spain \\ ${ }^{3}$ Laboratoire de Physique Quantique, IRSAMC, Université Paul Sabatier, 118, route de Narbonne, F-31062 Toulouse Cedex, France \\ ${ }^{4}$ Departament de Química Física i Inorgànica and Institut d'Estudis Avançats, Universitat Rovira $i$ Virgili, \\ Plaça Imperial Tarraco 1, E-43005 Tarragona, Spain \\ ${ }^{5}$ Departament de Química Orgànica, Universitat de Barcelona, Martí i Franquès 1, E-08028 Barcelona, Spain
}

(Received 23 April 2002; published 31 October 2002)

The foundations and limits of $S=\frac{1}{2}$ and $S=1$ spin Hamiltonians for systems with two unpaired electrons in two well-defined orbitals per site are discussed by merging accurate ab initio calculations in binuclear systems with the effective Hamiltonian theory. It is shown that, beyond the usual $J_{i j} \hat{S}_{i} \cdot \hat{S}_{j}$ terms, the effective spin Hamiltonian necessarily introduces four-body spin operators in the $S=\frac{1}{2}$ case and biquadratic terms in the $S$ $=1$ formalism. The order of magnitude of these additional terms can be rationalized from a quasidegenerate perturbation theory expansion starting from a Hubbard-type Hamiltonian. This permits to discuss the physical mechanisms governing the reduction from the all electron Hamiltonian to the spin-only Hamiltonians and the conditions under which a further reduction from a spin Hamiltonian to the simplest Heisenberg-Dirac-Van Vleck form is possible. The overall discussion is illustrated by numerical calculations of the magnetic coupling between two $\mathrm{Ni}^{2+}$ cations in the $\mathrm{K}_{2} \mathrm{NiF}_{4}$ perovskite and between triply bonded carbon atoms in poly-ynes.

DOI: 10.1103/PhysRevB.66.134430

PACS number(s): 75.10.Dg

\section{INTRODUCTION}

Magnetic systems with localized spin moments can be described by concentrating on the unpaired electrons in the field provided by the rest of electrons and the nuclear framework. The dynamical response of the core electrons to the fluctuation of the unpaired electron density may be crucial for the quantitative calculation of the intersite magnetic coupling constant; but for the modeling of those properties involving only the magnetic sites, total spin can be effectively included in the resulting parameters. The mathematical formulation of this simple model follows the well-known Heisenberg-Dirac-Van Vleck HDVV Hamiltonian ${ }^{1,2}$

$$
\hat{H}^{\mathrm{HDVV}}=-\sum_{\langle i, j\rangle} J_{i j} \hat{S}_{i} \cdot \hat{S}_{j},
$$

where $J_{i j}\left(J_{i j}>0\right.$ for a ferromagnetic interaction $)$ is the magnetic coupling constant governing the energy difference between the different spin states, $\hat{S}_{i}$ and $\hat{S}_{j}$ are the total spin operators for centers $i$ and $j$ and the symbol $\langle i, j\rangle$ indicates summation over all $i$ and $j$ neighbor magnetic centers. For systems in which each site only contributes with one unpaired electron in a well-defined and localized magnetic orbital, the status of the HDVV Hamiltonian is rather clear. ${ }^{3}$ It can be seen, as derived from the exact Hamiltonian through the application of the effective Hamiltonian theory, ${ }^{4-6}$ when the model space is spanned by the neutral valence bond (VB) determinants. ${ }^{7,8}$ These neutral VB determinants are simply those with a common closed-shell core and with all magnetic orbitals singly occupied. Since all determinants in the model space have the same space part, only differing by the spin distribution, the effective Hamiltonian is a spin-only Hamiltonian. However, this does not prove that the resulting spin
Hamiltonian reduces to the simple form of Eq. (1). The pioneering work of Nesbet ${ }^{9-11}$ suggested that two particles with spin $S$ should interact according to the Heisenberg Hamiltonian. However, the detailed analysis of Herring ${ }^{3}$ shows that the generalization is not exact and that higher-order terms appear in a more elaborate description. A similar conclusion can be reached by simply starting from the well-known Hubbard Hamiltonian

$$
\hat{H}^{\text {Hubbard }}=-\sum_{\langle i, j\rangle} t_{i j}\left(\hat{a}_{i}^{\dagger} \hat{a}_{j}+\hat{a}_{j}^{\dagger} \hat{a}_{i}\right)+U \sum_{i} \hat{a}_{i \alpha}^{\dagger} \hat{a}_{i \alpha} \hat{a}_{i \beta}^{\dagger} \hat{a}_{i \beta},
$$

where $t_{i j}$ is the intersite hopping integral, $U$ the on-site effective two-electron repulsion and the $\hat{a}_{i}^{\dagger}$ and $\hat{a}_{i}$ the usual creation and annihilation quasiparticle operators. To the second order of perturbation theory only two-body interactions appear and it is rather easy to show that the magnetic coupling constant takes the simple form

$$
J_{i j}=-\frac{4 t_{i j}^{2}}{U},
$$

However, this is only a second-order development. At the fourth-order expansion, four-body operators appear by permuting all spins in a four-member ring ${ }^{12-14}$ for instance, a square or rectangular plaquette. These four-body operators can be formally written as

$$
K_{4}\left[\left(\hat{S}_{i} \cdot \hat{S}_{j}\right)\left(\hat{S}_{k} \cdot \hat{S}_{l}\right)+\left(\hat{S}_{j} \cdot \hat{S}_{k}\right)\left(\hat{S}_{l} \cdot \hat{S}_{i}\right)-\left(\hat{S}_{i} \cdot \hat{S}_{k}\right)\left(\hat{S}_{j} \cdot \hat{S}_{l}\right)\right],
$$




$$
K_{4}=\frac{80 t_{i j}^{4}}{U^{3}}
$$

is a quantity that, in many circumstances, is not negligible. ${ }^{12}$

Embedded clusters have long been proposed as suitable models of bulk ionic systems and, in particular, to extract electronic structure parameters from pertinent $a b$ initio calculations. Thirty years ago, Wachters and Nieuwpoort employed a cluster model representation of $\mathrm{KNiF}_{3}$ to compute the magnetic coupling constant of this material. ${ }^{15}$ Following these ideas Chen and Goddard ${ }^{16}$ and Martin ${ }^{17,18}$ studied the electronic structure and magnetic coupling in $\mathrm{La}_{2} \mathrm{CuO}_{4}$ using wave-function based methods. A similar cluster model approach was used by Hybertsen and co-workers to derive electronic structure parameters, ${ }^{19-21}$ although these authors relied on the local-density approach (LDA) to densityfunctional theory (DFT). Here, we must advert that subsequent work has shown that DFT and, in particular, LDA, do not provide an adequate description of the electronic structure of these strongly correlated systems, ${ }^{22-25}$ whereas configuration-interaction techniques can provide an accurate description of the local electronic structure parameters. ${ }^{26,27}$ For a broad series of ionic systems, including high- $T_{c}$ superconductors, it has been shown that $J_{i j}$ can be accurately determined by means of configuration-interaction calculations carried out in embedded cluster models. ${ }^{28-36}$ This approach has been extended and permitted to obtain the hopping integral and magnetic coupling constant of monolayered cuprate superconductors ${ }^{37}$ revealing the existence of a quantitative relationship between the measured $T_{c}$ and the calculated $J / t$ ratio. ${ }^{38,39}$ Recently, the application of the effective Hamiltonian theory together with the embedded cluster approach has also permitted to obtain $\mathrm{K}_{4}$ for $\mathrm{La}_{2} \mathrm{CuO}_{4} \cdot{ }^{14}$ For this superconductor parent compound the $K_{4} / J$ ratio appears to be $\sim 0.1$ indicating a small but noticeable deviation from the simple HDVV Hamiltonian. In six-membered rings-for instance, the $\pi$ system of benzene and graphitic honeycomb lattices-a similar six-body operator appears at the sixth order of the perturbation expansion, the amplitude of which is $504 t_{i j}^{6} / U^{5} .{ }^{12}$ Clearly, the simple usual form of the HDVV Hamiltonian is only a low-order approximation, frequently sufficient in practice, of the exact effective Hamiltonian which may be derived from the exact Hamiltonian in a similar way as discussed above for the Hubbard model Hamiltonian.

The present paper concentrates on systems where each magnetic site bears two unpaired electrons, in well-defined and localized singly occupied (or magnetic) orbitals. This is the case of the $\mathrm{Ni}^{2+}$ cations in $\mathrm{NiO}$ or $\mathrm{K}_{2} \mathrm{NiF}_{4}$. In both cases the crystal field removes the atomic symmetry, and six of the eight $d$ electrons are accommodated in a $t_{2 g}$ closed shell and the other two electrons occupy the $e_{g}$ shell, which remains half filled. In these systems the crystal field fixes the orientation of the two unpaired electrons in well-defined atomiclike orbitals. One might also consider sp-hybridized carbon atoms involved in the $\mathrm{C}-\mathrm{C}$ triple bond of poly-ynes. In these polymers each carbon atom contributes with two electrons, each of them participating in one of the two orthogonal $\pi$ systems.
Considering the simplest architecture, namely, a twomagnetic site system with four unpaired electrons, the present paper analyzes the possible foundation of two types of Heisenberg Hamiltonians to describe the low-lying spectrum of such a system. Those are a $S=\frac{1}{2}$ Heisenberg Hamiltonian involving four spins in four orbitals and the usual $S$ $=1$ Heisenberg Hamiltonian, which assumes that the two electrons on a magnetic site are ferromagnetically coupled in an atomic triplet state. In the former case it can be shown that a four-orbital operator appears in the effective Hamiltonian, whereas in the latter a biquadratic term appears and the effective HDVV Hamiltonian becomes

$$
\hat{H}^{\mathrm{HDVV}}=-\sum_{\langle i, j\rangle} J_{i j}\left\{\hat{S}_{i} \cdot \hat{S}_{j}+\lambda\left(\hat{S}_{i} \cdot \hat{S}_{j}\right)^{2}\right\}
$$

This paper is organized as follows. In Sec. II we first briefly review the definition of the exact effective Hamiltonian from the exact spectrum, according to Bloch's and des Cloizeaux's original theory. ${ }^{40,41}$ Section III defines the three possible model spaces whereas the structure of the corresponding effective Hamiltonian together with the formal and logical aspects of the problem associated with the definition of the different $S=\frac{1}{2}$ and $S=1$ model spaces are discussed in Sec. IV. In Sec. V, the fourth-order expansion of the two spin Hamiltonians discussed above is derived from a Hubbardlike Hamiltonian. This derivation permits to discuss the relative importance, i.e., the amplitude, of the fourth-order terms. Finally, Sec. VI presents an effective Hamiltonian for $\mathrm{K}_{2} \mathrm{NiF}_{4}$ derived from accurate configuration-interaction calculations on an embedded-cluster representation of this compound. ${ }^{31}$ This analysis shows that the four-body and biquadratic terms are indeed small but obey the algebraic relations analytically established in Secs. II-IV. A similar analysis is presented for ethyne, $\mathrm{H}-\mathrm{C} \equiv \mathrm{C}-\mathrm{H}$. In this molecule the monocentric ferromagnetic exchange is much smaller than in $\mathrm{K}_{2} \mathrm{NiF}_{4}$, while the effective intersite hopping integral is larger. Using again accurate configuration-interaction wave functions, the $S=\frac{1}{2}$ and $S=1$ spin Hamiltonians have been derived. In this case the four-body operators and biquadratic terms have very large amplitudes.

\section{RUDIMENTS OF EFFECTIVE HAMILTONIAN THEORY}

For a system with a given exact Hamiltonian the mathematical structure of quantum mechanics ensures that there is a complete set of eigenfunctions satisfying the timeindependent Schrödinger equation

$$
\hat{H}\left|\Psi_{m}\right\rangle=E_{m}\left|\Psi_{m}\right\rangle .
$$

Usually, one is not interested in the whole spectrum but rather in a small number $M$ of states, defining a target space through its proper projector operator, simply defined as

$$
\hat{P}_{\text {target }}=\sum_{i=1, M}\left|\Psi_{i}\right\rangle\left\langle\Psi_{i}\right|
$$


According to Bloch's and des Cloizeaux's theory ${ }^{40,41}$ (see also Refs. 5 and 6) it is possible to define an isodimensional model space $S$ of projector

$$
\hat{P}=\sum_{i=1, M}\left|\Phi_{i}\right\rangle\left\langle\Phi_{i}\right|
$$

and an exact effective Hamiltonian acting on the model space and such that the $M$ eigenvalues match exactly those of the exact Hamiltonian and the $M$ eigenfunctions are the projections of the exact wave functions onto the model space,

$$
\hat{H}^{\mathrm{eff}}\left|\hat{P} \Psi_{m}\right\rangle=E_{m}\left|\hat{P} \Psi_{m}\right\rangle, \quad m=1, M .
$$

Clearly, $\hat{H}^{\text {eff }}$ only permits to recover those states having significant projections onto the model space. The $M$ equations (10) impose $M+M(M-1)$ conditions, i.e., they uniquely define the $M^{2}$ matrix elements of $\hat{H}^{\text {eff }}$.

The spectral definition of $\hat{H}^{\text {eff }}$ provides a simple and computationally convenient way to represent this effective operator. This is given by

$$
\hat{H}^{\mathrm{eff}}=\sum_{m=1, M}\left|\hat{P} \Psi_{m}\right\rangle E_{m}\left\langle\hat{P} \Psi_{m}^{\perp}\right|,
$$

where $\left|\hat{P} \Psi_{m}^{\perp}\right\rangle$ is the biorthogonal vector associated with $\left|\hat{P} \Psi_{m}\right\rangle$. In principle, while the different state vectors fulfilling Eqs. (7) are orthogonal, there is no reason, except for possible symmetry arguments, for the projections of these states onto the model space to be orthogonal. Nevertheless, it is always possible to orthogonalize these projections as suggested by des Cloizeaux ${ }^{41}$ and the corresponding effective Hamiltonian is indeed Hermitian. Of course, the resulting effective Hamiltonian depends on the choice of the $M$ states defining the target space.
The effective Hamiltonian is related to the so-called wave operator, $\Omega$, allowing to obtain the exact wave function from its projection on the lower-dimensional model space,

$$
\begin{gathered}
\hat{P}_{\text {target }}=\Omega \hat{P} \\
\text { or } \quad \hat{H}^{\text {eff }}=\hat{P} \hat{H} \Omega \hat{P} ;
\end{gathered}
$$

the best choice of the target space is the one minimizing the norm of the wave operator

$$
\|\Omega\|=\min ,
$$

and it is spanned by the $M$ eigenstates having the largest linear independent projection onto the model space

$$
\sum_{i=1, M}\left\|\hat{P} \Psi_{i}\right\|=\max
$$

From Eq. (11) it is clear that the effective Hamiltonian is uniquely defined by the choice of the model space and the knowledge of the target space eigenvectors and eigenenergies. However, the identification of the relevant $M$ eigenstates satisfying this condition is not always straightforward. If the model space involves determinants of high energy, they will appear with large coefficients in high-energy eigenstates, they are frequently spread on a broad range of eigenstates and the definition of the target space may become impossible.

When the eigenenergies and the eigenvectors of $\hat{H}$ are not known, it is possible to build the effective Hamiltonian from the model space through an order-by-order expansion, according to the quasidegenerate perturbation theory. If this expansion converges, it leads to the exact effective Hamiltonian. The low-order terms are

$$
\begin{aligned}
\left\langle\Phi_{I}\left|\hat{H}^{\mathrm{eff}}\right| \Phi_{J}\right\rangle= & \left\langle\Phi_{I}|\hat{V}| \Phi_{J}\right\rangle+\sum_{\alpha \notin S} \frac{\left\langle\Phi_{I}|\hat{V}| \Phi_{\alpha}\right\rangle\left\langle\Phi_{\alpha}|\hat{V}| \Phi_{J}\right\rangle}{E_{J}^{0}-E_{\alpha}^{0}} \\
& +\sum_{\alpha \notin S} \sum_{\beta \notin S} \frac{\left\langle\Phi_{I}|\hat{V}| \Phi_{\alpha}\right\rangle\left\langle\Phi_{\alpha}|\hat{V}| \Phi_{\beta}\right\rangle\left\langle\Phi_{\beta}|\hat{V}| \Phi_{J}\right\rangle}{\left(E_{J}^{0}-E_{\alpha}^{0}\right)\left(E_{J}^{0}-E_{\beta}^{0}\right)}-\sum_{K \in S} \sum_{\alpha \notin S} \frac{\left\langle\Phi_{I}|\hat{V}| \Phi_{\alpha}\right\rangle\left\langle\Phi_{\alpha}|\hat{V}| \Phi_{K}\right\rangle\left\langle\Phi_{K}|\hat{V}| \Phi_{J}\right\rangle}{\left(E_{J}^{0}-E_{a}^{0}\right)\left(E_{K}^{0}-E_{\alpha}^{0}\right)} \\
& +\sum_{\alpha \notin S} \sum_{\beta \notin S} \sum_{\gamma \notin S} \frac{\left\langle\Phi_{I}|\hat{V}| \Phi_{\alpha}\right\rangle\left\langle\Phi_{\alpha}|\hat{V}| \Phi_{\beta}\right\rangle\left\langle\Phi_{\beta}|\hat{V}| \Phi_{\gamma}\right\rangle\left\langle\Phi_{\gamma}|\hat{V}| \Phi_{J}\right\rangle}{\left(E_{J}^{0}-E_{\alpha}^{0}\right)\left(E_{J}^{0}-E_{\beta}^{0}\right)\left(E_{J}^{0}-E_{\gamma}^{0}\right)} \\
& -\sum_{\alpha \notin S} \sum_{\gamma \notin S} \sum_{K \in S} \frac{\left\langle\Phi_{I}|\hat{V}| \Phi_{\alpha}\right\rangle\left\langle\Phi_{\alpha}|\hat{V}| \Phi_{K}\right\rangle\left\langle\Phi_{K}|\hat{V}| \Phi_{\gamma}\right\rangle\left\langle\Phi_{\gamma}|\hat{V}| \Phi_{J}\right\rangle}{\left(E_{J}^{0}-E_{\alpha}^{0}\right)^{2}\left(E_{J}^{0}-E_{\gamma}^{0}\right)} \\
& -\sum_{\alpha \notin S} \sum_{\beta \notin S} \sum_{K \in S} \frac{\left\langle\Phi_{I}|\hat{V}| \Phi_{\alpha}\right\rangle\left\langle\Phi_{\alpha}|\hat{V}| \Phi_{\beta}\right\rangle\left\langle\Phi_{\beta}|\hat{V}| \Phi_{K}\right\rangle\left\langle\Phi_{K}|\hat{V}| \Phi_{J}\right\rangle}{\left(E_{J}^{0}-E_{\alpha}^{0}\right)\left(E_{J}^{0}-E_{\beta}^{0}\right)}\left(\frac{1}{\left.E_{K}^{0}-E_{\alpha}^{0}+\frac{1}{E_{K}^{0}-E_{\beta}^{0}}\right)}\right. \\
& +\sum_{\alpha \notin S} \sum_{K \in S} \sum_{L \in S} \frac{\left\langle\Phi_{I}|\hat{V}| \Phi_{\alpha}\right\rangle\left\langle\Phi_{\alpha}|\hat{V}| \Phi_{L}\right\rangle\left\langle\Phi_{L}|\hat{V}| \Phi_{K}\right\rangle\left\langle\Phi_{K}|\hat{V}| \Phi_{J}\right\rangle}{\left(E_{J}^{0}-E_{\alpha}^{0}\right)\left(E_{K}^{0}-E_{\alpha}^{0}\right)\left(E_{L}^{0}-E_{\alpha}^{0}\right)}
\end{aligned}
$$


where $\hat{V}$ is the appropriate perturbation operator, i.e., $\hat{V}=\hat{H}$ $-\hat{H}_{0}$, and $\hat{H}_{0}$ a convenient choice of the zero-order Hamiltonian. ${ }^{4-7}$

The perturbation expansion diverges if some of the outer space determinants, $\left|\Phi_{\alpha}\right\rangle$, are close in energy to the highenergy model space determinants; this is the well-known intruder state problem. ${ }^{42}$ There is a logical connection between the above-mentioned possible ambiguities in the choice of the target eigenvectors and the divergences in the quasidegenerate perturbation theory (QDPT) expansion. Nevertheless, the QDPT is essentially a tool for analysis when starting from a simple model Hamiltonian such as the Hubbard Hamiltonian.

\section{THE THREE POSSIBLE DEFINITIONS OF THE MODEL SPACE}

In the preceding section it has been recalled that an effective Hamiltonian projects a part of the physics of a system, which is described in its corresponding Hilbert space, onto a low-dimensional model space. For those systems in which the physics can be reduced to a set of particles with total spin $\frac{1}{2}$, i.e., electrons, with only one particle and one orbital per site, the model space is unambiguous. The lowest-energy eigenstates are given by linear combinations of all the Slater determinants that can be built by distributing all the electrons in all the magnetic orbitals with the restriction of avoiding double occupancy. The space part of all these determinants is the same. Therefore, the different Slater determinants differ only by their spin distribution and, as a result, the effective Hamiltonian can only be a spin Hamiltonian. Moreover, since the space part is made of atomic orbitals, the Slater determinants defined above can be viewed as neutral VB determinants since they all have the common feature of keeping one electron per site.

In the case of two particles per site, the situation is rather more complex. In fact, for an atom $A$ contributing with two unpaired electrons, described by the atomic, well-defined, magnetic orbitals $a$ and $a^{\prime}$, several two-electron distributions maintain the atomic neutrality and have an unambiguous spin state, namely, the three components of the lowestenergy triplet state $T$ and three singlet states $S$. The configuration functions defining the triplet state can be schematically represented by

$$
\begin{gathered}
T_{A}^{0}=\frac{1}{\sqrt{2}}\left\{\operatorname{det}\left(a \bar{a}^{\prime}\right)+\operatorname{det}\left(\bar{a} a^{\prime}\right)\right\}, \\
T_{A}^{+}=\operatorname{det}\left(a a^{\prime}\right), \\
T_{A}^{-}=\operatorname{det}\left(\bar{a} \bar{a}^{\prime}\right) .
\end{gathered}
$$

Similarly the three singlet states may be written as

$$
\begin{aligned}
& S_{A}=\frac{1}{\sqrt{2}}\left\{\operatorname{det}\left(a \bar{a}^{\prime}\right)-\operatorname{det}\left(\bar{a} a^{\prime}\right)\right\}, \\
& S_{A^{\prime}}=\frac{1}{\sqrt{2}}\left\{\operatorname{det}(a \bar{a})-\operatorname{det}\left(a^{\prime} \bar{a}^{\prime}\right)\right\}, \\
& S_{A^{\prime \prime}}=\frac{1}{\sqrt{2}}\left\{\operatorname{det}(a \bar{a})+\operatorname{det}\left(a^{\prime} \bar{a}^{\prime}\right)\right\},
\end{aligned}
$$

where $a$ and $\bar{a}$ stand for the $\alpha$ and $\beta$ spin, respectively. Defining the product of the irreducible representations of the orbitals $a$ and $a^{\prime}$ as $D$ (in the atom symmetry group notation, when $a$ and $a^{\prime}$ are $p$-type orbitals), it is straightforward to show that the triplet states are all of $D$ symmetry and have total spin $z$ component, $M_{S}=0,1,-1$. Likewise, for the singlet states two different symmetries are possible; $S_{A}$ is an open-shell singlet of $D$ symmetry or ${ }^{1} D, S_{A^{\prime}}$ a closed-shell singlet of the same symmetry (also ${ }^{1} D$ ), and $S_{A^{\prime \prime}}$ a closedshell singlet of $S$ symmetry or ${ }^{1} S$.

Defining $K$ as the (positive) atomic exchange integral,

$$
K=\left\langle a a^{\prime}|\hat{H}| a^{\prime} a\right\rangle=\left\langle a a^{\prime}\left|\frac{1}{r_{12}}\right| a^{\prime} a\right\rangle,
$$

it is easy to show, from atomic spectroscopy, that the energy difference between the two-electron Coulomb repulsion integrals, $J^{\text {Coulomb }}$, for the two electrons in the orbital $a$ or that for one electron in $a$ and one in $a^{\prime}$ is

$$
J_{a a}^{\text {Coulomb }}-J_{a a^{\prime}}^{\text {Coulomb }}=\left\langle a a\left|\frac{1}{r_{12}}\right| a a\right\rangle-\left\langle a a^{\prime}\left|\frac{1}{r_{12}}\right| a a^{\prime}\right\rangle=2 K,
$$

and hence the energy difference between the two degenerate singlet states of $D$ symmetry, $S_{A}$ and $S_{A^{\prime}}$, and the lowest triplet is $2 K$, while the ${ }^{1} S$ state lies $3 K$ above the triplet, i.e.,

$$
\begin{aligned}
& E_{1_{D}}=E_{T_{A}^{0}}+2 K, \\
& E_{1_{S}}=E_{T_{A}^{0}}+3 K .
\end{aligned}
$$

Different model spaces may be considered for the $A B$ system, all of them keeping two electrons on each atom but of smaller and smaller dimension. The largest model space would keep all the neutral, $A^{0} B^{0}, \mathrm{VB}$ determinants. If the orbitals $a$ and $b$ are of different symmetry than $a^{\prime}$ and $b^{\prime}$, the states with one (or three) electron in the subset $\{a, b\}$ and three (or one) in the subset $\left\{a^{\prime}, b^{\prime}\right\}$ are of different symmetry than the states keeping two electrons in each subset. Hereafter, we will only consider the latter family, which generates the lowest eigenstates of the system. For convenience, we concentrate on the $M_{S}=0$ manifold, which involves ten determinants, schematized in Fig. 1. The orientation of the magnetic orbitals symbolizes their respective symmetries. Among them, six avoid double occupancy, namely, 


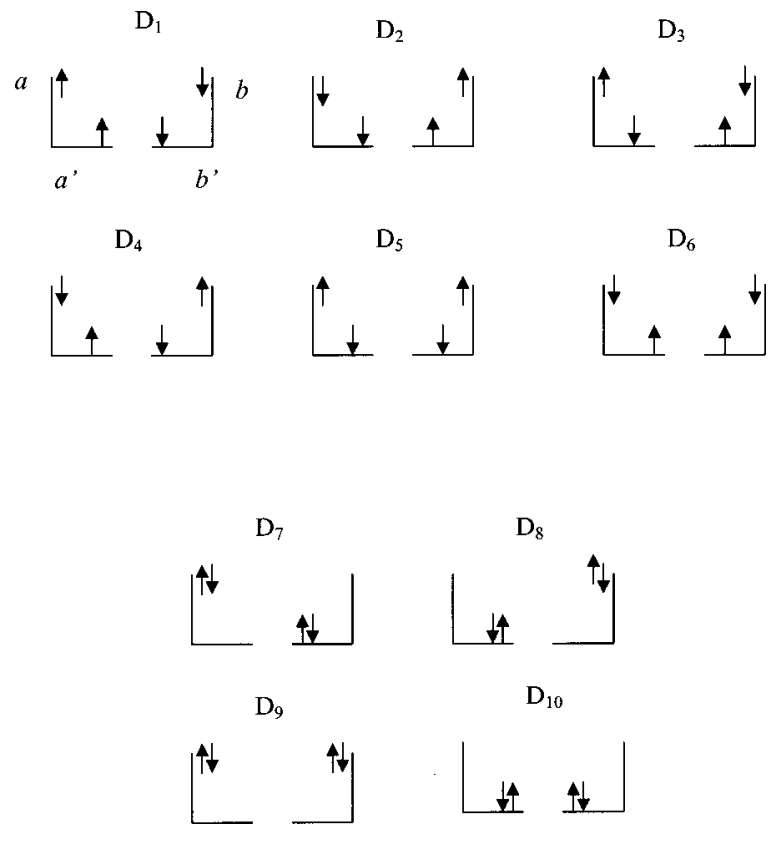

FIG. 1. Schematic representation of the ten neutral VB determinants defined in an $A B$ dimer by two magnetic orbitals ( $a$ and $a^{\prime}$ or $b$ and $\left.b^{\prime}\right)$ per center. The relative orientation aims to mimic the different symmetry of the magnetic orbitals.

$$
\begin{gathered}
D_{1}=\operatorname{det}\left(a a^{\prime} \bar{b} \bar{b}^{\prime}\right)=T_{A}^{+} T_{B}^{-}, \\
D_{2}=\operatorname{det}\left(\bar{a} \bar{a}^{\prime} b b^{\prime}\right)=T_{A}^{-} T_{B}^{+}, \\
D_{3}=\operatorname{det}\left(a \bar{a}^{\prime} \bar{b} b^{\prime}\right), \\
D_{4}=\operatorname{det}\left(\bar{a} a^{\prime} b \bar{b}^{\prime}\right), \\
D_{5}=\operatorname{det}\left(a \bar{a}^{\prime} b \bar{b}^{\prime}\right), \\
D_{6}=\operatorname{det}\left(\bar{a} a^{\prime} \bar{b} b^{\prime}\right) .
\end{gathered}
$$

The $D_{1}$ and $D_{2}$ determinants are of lower energy since they satisfy the intra-atomic Hund's rule. They are coupled to the ionic $A^{-} B^{+}$and $A^{+} B^{-} \mathrm{VB}$ determinants, of the $\operatorname{det}\left(a \bar{a} a^{\prime} \bar{b}^{\prime}\right)$ or $\operatorname{det}\left(a b \bar{b} b^{\prime}\right)$ type. This coupling gives rise to the so-called intersite antiferromagnetic kinetic exchange. ${ }^{43}$ These ionic VB determinants also interact with the determinants $D_{3}$ and $D_{4}$, which violate the intra-atomic Hund's rule. The role of $D_{3}$ and $D_{4}$ in the lowest singlet wave function should be less important than that of $D_{1}$ and $D_{2}$. Finally, in $D_{5}$ and $D_{6}$, which also violate the intra-atomic Hund's rule, intersite hopping cannot take place. Hence, the weight of these determinants in the lowest singlet eigenstate should be even smaller than those of the previous ones. Schematically one has $\left|C_{5,6}\right|<\left|C_{3,4}\right|<\left|C_{1,2}\right|$, where $C_{i}$ is the coefficient of the determinant $D_{i}$ in the wave-function expansion. The four remaining determinants that complete the neutral VB model space are

$$
\begin{gathered}
D_{7}=\operatorname{det}\left(a \bar{a} b^{\prime} \bar{b}^{\prime}\right), \\
D_{8}=\operatorname{det}\left(a^{\prime} \bar{a}^{\prime} b \bar{b}\right), \\
D_{9}=\operatorname{det}(a \bar{a} b \bar{b}), \\
D_{10}=\operatorname{det}\left(a^{\prime} \bar{a}^{\prime} b^{\prime} \bar{b}^{\prime}\right) .
\end{gathered}
$$

$D_{7}$ and $D_{8}$ have double occupancy of the magnetic orbitals but they are directly coupled to the singly ionic $A^{+} B^{-}$and $A^{-} B^{+}$determinants, i.e., they accept instantaneous interatomic delocalization. Such a delocalization is impossible for $D_{9}$ and $D_{10}$, which should have a much weaker contribution to the lowest eigenstates.

The ten determinants discussed above do not have the same space part. Working with such a ten-dimensional model space would lead to an effective Hamiltonian handling both the spin and orbital variables and, consequently, it will not be a spin (or HDVV) Hamiltonian. Moreover, the determinants $D_{9}$ and $D_{10}$ are so high in energy that they will appear with large coefficients in a broad set of high-energy eigenstates. For such a model space the identification of the target space would be impossible or arbitrary. Accordingly, this model space will not be considered in further discussion.

On the other hand, the first six determinants, Eq. (22), having a common space part define a six-dimensional model space leading to a spin-only effective Hamiltonian. Here, there are four particles with $S=\frac{1}{2}$ and the effective Hamiltonian is an $S=\frac{1}{2}$ spin Hamiltonian with possible four-body operators. One drawback of this model space is that it is not invariant under on-site rotation of the magnetic orbitals. A unitary transformation of the magnetic orbitals $a$ and $a^{\prime}$ leads to

$$
\begin{gathered}
\tilde{a}=a \cos \varphi+a^{\prime} \sin \varphi, \\
\widetilde{a}^{\prime}=-a \sin \varphi+a^{\prime} \cos \varphi,
\end{gathered}
$$

and since

$$
\widetilde{a} \overline{\widetilde{a}}^{\prime}=\left(\cos ^{2} \varphi\right) a \bar{a}^{\prime}-\left(\sin ^{2} \varphi\right) a^{\prime} \bar{a}+\sin \varphi \cos \varphi\left(a^{\prime} \bar{a}^{\prime}-a \bar{a}\right),
$$

the new open-shell distribution involves part of the previous closed-shell ones. Nevertheless, there are two reasons to keep this model space. First, the environment of the $A B$ magnetic centers may provide a natural choice for the magnetic orbitals, e.g., the $\mathrm{Cu}\left(d_{x^{2}-y^{2}}\right)$ in the $\mathrm{CuO}$ planes of high- $T_{c}$ superconducting cuprates. Second, this model space allows to enlighten the relationships between the four-body and the biquadratic operators in the $S=\frac{1}{2}$ and $S=1$ spin Hamiltonians. But $D_{7}$ and $D_{8}$ are neutral and coupled to the singly ionic VB resonating structures and they may be responsible for strong fourth-order effects in the perturbative expansion from the six-dimensional model space and eventually act as intruder states.

The third (three-dimensional) model space consists in considering only the states that arise from the products of local triplets defined in Eq. (17). These are $D_{1}$ and $D_{2}$ plus 
TABLE I. Matrix representation of the effective Hamiltonian in the $S=\frac{1}{2}$ model space, using the $D_{1}$ to $D_{6}$ basis defined in Eq. (22).

\begin{tabular}{lccccc}
\hline \hline$\hat{H}^{\text {eff }}$ & $D_{1}$ & $D_{2}$ & $D_{3}$ & $D_{4}$ & $D_{5}$ \\
\hline$D_{1}$ & $\left(j+j^{\prime}+2 j^{\prime \prime}\right) / 2-h$ & & & & \\
$D_{2}$ & $h$ & $\left(j+j^{\prime}+2 j^{\prime \prime}\right) / 2-h$ & & & \\
$D_{3}$ & $-j^{\prime} / 2$ & $-j / 2$ & $2 K+\left(j+j^{\prime}\right) / 2-h^{\prime}$ & & \\
$D_{4}$ & $-j / 2$ & $-j^{\prime} / 2$ & $h^{\prime}$ & $2 K+\left(j+j^{\prime}\right) / 2-h^{\prime}$ & $2 K+j^{\prime \prime}-h^{\prime \prime}$ \\
$D_{5}$ & $-j^{\prime \prime} / 2$ & $-j^{\prime \prime} / 2$ & $-K$ & $-K$ & $h^{\prime \prime}$ \\
$D_{6}$ & $-j^{\prime \prime} / 2$ & $-j^{\prime \prime} / 2$ & $-K$ & $-K$ & $2 K+j^{\prime \prime}-h^{\prime \prime}$ \\
\hline \hline
\end{tabular}

$$
\begin{aligned}
D_{3}^{\prime} & =\frac{1}{2}\left[\left\{\operatorname{det}\left(a \bar{a}^{\prime}\right)+\operatorname{det}\left(\bar{a} a^{\prime}\right)\right\}\left\{\operatorname{det}\left(b \bar{b}^{\prime}\right)+\operatorname{det}\left(\bar{b} b^{\prime}\right)\right\}\right] \\
& =T_{A}^{0} T_{B}^{0} .
\end{aligned}
$$

This model space generates an $S=1$ spin Hamiltonian. Notice that, since

$$
D_{3}^{\prime}=\frac{1}{2}\left(D_{3}+D_{4}+D_{5}+D_{6}\right),
$$

the contribution of determinants $D_{5}$ and $D_{6}$ is the same as that of determinants $D_{3}$ and $D_{4}$, which are expected to have a larger contribution in the lowest-energy eigenstates.

\section{LOGICAL FORMS OF THE $S=\frac{1}{2}$ AND $S=1$ SPIN HAMILTONIANS}

In this section we discuss the analytical form of the effective Hamiltonian matrix elements corresponding to the model spaces presented in the preceding section. For convenience, the $S=\frac{1}{2}$ and $S=1$ spin Hamiltonians will be discussed separately.

\section{A. The spin Hamiltonian in the $S=\frac{1}{2}$ model space}

The six-dimensional model space, spanned by the determinants $D_{1}$ to $D_{6}$ generates a Hermitian effective Hamiltonian defined by $6 \times \frac{5}{2}=15$ parameters. The corresponding eigenstates can be classified according to the spin, and eventually space, symmetry. There are two singlet stateshereafter referred to as $S_{1}$ and $S_{2}$, three triplet states $-T_{1}$, $T_{2}$, and $T_{3}$, and one quintet state- $Q$; i.e., five energy differences. Let us consider the degrees of freedom in the coefficients of the eigenstates that must already satisfy spin and space symmetry requirements as well as the normalization constraint. In a homonuclear system, the states are of $g$ or $u$ symmetry. The two singlets and two triplets states are of $g$ symmetry (see below). If a Hermitian effective Hamiltonian is searched, both singlet states are orthogonal and the same is true for the two $g$ triplet states. These constraints lead to a unique degree of freedom for the coefficients of the two singlets and for those of the two triplet states of $g$ symmetry. There is no degree of freedom for the quintet nor for the $u$ triplet. The total number of degrees of freedom is therefore equal to seven. Actually the effective Hamiltonian is univocally defined by seven parameters. The matrix representation of this effective Hamiltonian, in the basis of the $D_{1}$ to $D_{6}$ determinants, is schematically shown in Table I. The meaning of the parameters defining this effective Hamiltonian is as follows (see scheme 1).

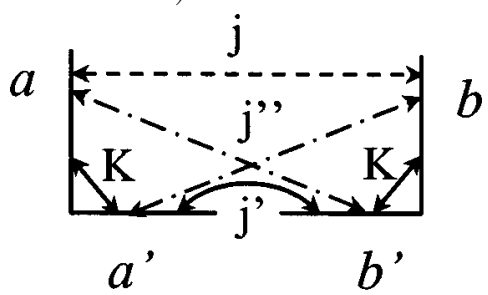

\section{Scheme 1}

(i) $j$ is the magnetic coupling constant involving the $a$ and $b$ magnetic orbitals centered on $A$ and $B$, respectively; $j$ $=J_{a b}$.

(ii) $j^{\prime}$ is the magnetic coupling constant involving the $a^{\prime}$ and $b^{\prime}$ magnetic orbitals centered on $A$ and $B$, respectively; $j^{\prime}=J_{a^{\prime} b^{\prime}}$.

(iii) $j^{\prime \prime}$ is the magnetic coupling constant involving the $a$ $\left(a^{\prime}\right)$ and $b^{\prime}(b)$ magnetic orbitals centered on $A$ and $B$, respectively; $j^{\prime \prime}=J_{a b^{\prime}}=J_{a^{\prime} b}$.

(iv) $h, h^{\prime}$, and $h^{\prime \prime}$ are related to effective four-body operators.

(v) $K$ is the on-site (ferromagnetic) effective exchange, cf. Eq. (19).

In the case where $a$ and $b^{\prime}$ and $a^{\prime}$ and $b$ are of different local symmetry, $j^{\prime \prime}$ is expected to be small. Likewise, for a system following the HDVV Hamiltonian the four-body operators should be negligible.

The structure of the effective Hamiltonian is much simpler when making use of the space and spin symmetries commented upon above. To this end, it is convenient to define the basis made of products of local (on-site) eigenstates,

$$
\begin{gathered}
D_{1}^{\prime}=T_{A}^{+} T_{B}^{-}=D_{1}, \\
D_{2}^{\prime}=T_{A}^{-} T_{B}^{+}=D_{2}, \\
D_{3}^{\prime}=T_{A}^{0} T_{B}^{0}=\frac{1}{2}\left\{D_{3}+D_{4}+D_{5}+D_{6}\right\}, \\
D_{4}^{\prime}=T_{A}^{0} S_{B}=\frac{1}{2}\left\{-D_{3}+D_{4}+D_{5}-D_{6}\right\}, \\
D_{5}^{\prime}=S_{A} T_{B}^{0}=\frac{1}{2}\left\{D_{3}-D_{4}+D_{5}-D_{6}\right\}, \\
D_{6}^{\prime}=S_{A} S_{B}=\frac{1}{2}\left\{-D_{3}-D_{4}+D_{5}+D_{6}\right\} .
\end{gathered}
$$

A subsequent simple transformation allows separating the singlet, triplet, and quintet subspaces leading to 
TABLE II. Matrix representation of the effective Hamiltonian in the $S=\frac{1}{2}$ model space using the space and spin adapted basis set defined in Eq. (29).

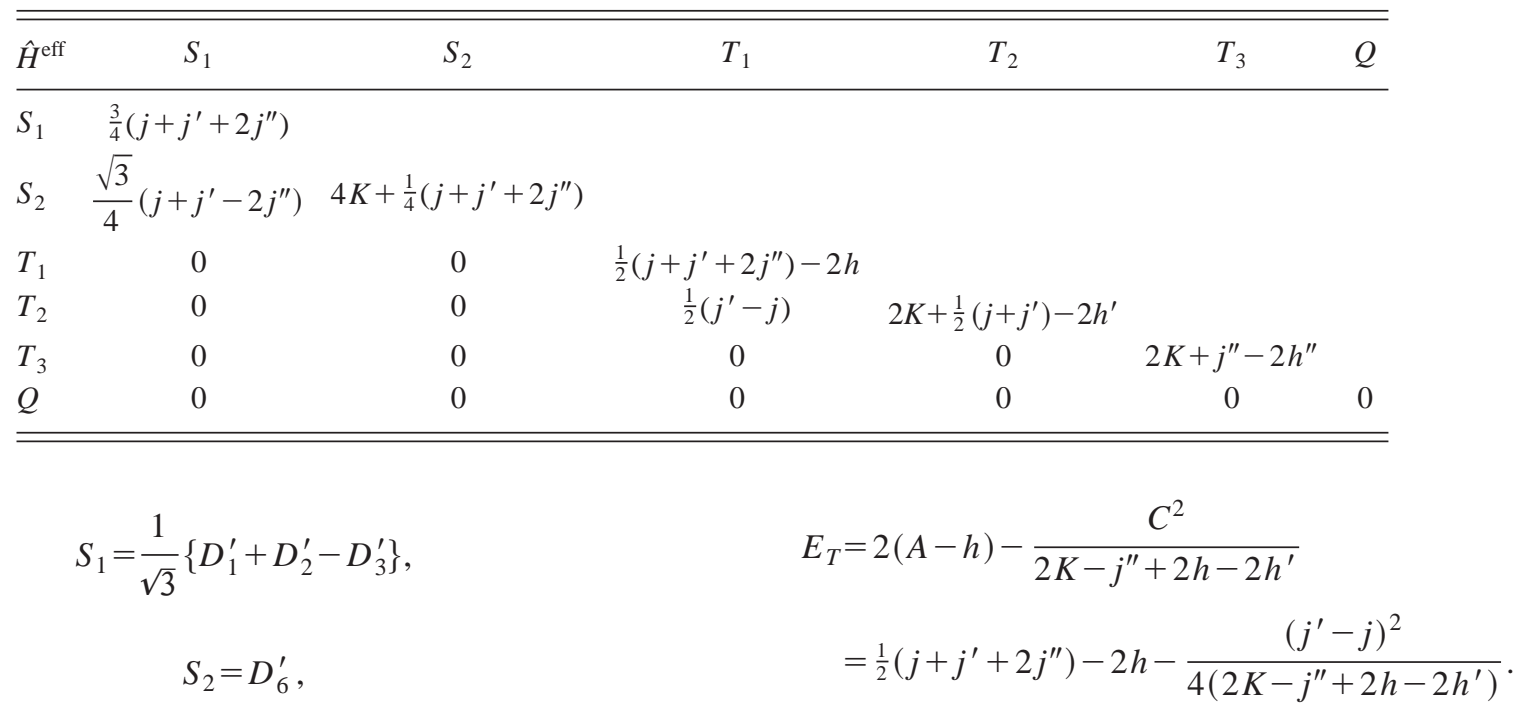

$$
\begin{gathered}
T_{1}=\frac{1}{\sqrt{2}}\left\{D_{1}^{\prime}-D_{2}^{\prime}\right\}, \\
T_{2}=\frac{1}{\sqrt{2}}\left\{D_{4}^{\prime}-D_{5}^{\prime}\right\}, \\
T_{3}=\frac{1}{\sqrt{2}}\left\{D_{4}^{\prime}+D_{5}^{\prime}\right\}, \\
Q=\frac{1}{\sqrt{6}}\left\{D_{1}^{\prime}+D_{2}^{\prime}+2 D_{3}^{\prime}\right\},
\end{gathered}
$$

and, since $T_{3}$ is of a different space symmetry than $T_{1}$ and $T_{2}$, the Hamiltonian takes the structure given in Table II, where the energy of the quintet state has been set for convenience to zero. By introducing

$$
\begin{gathered}
A=\frac{1}{4}\left(j+j^{\prime}+2 j^{\prime \prime}\right), \\
B=\frac{1}{4}\left(j+j^{\prime}-2 j^{\prime \prime}\right), \\
C=\frac{1}{2}\left(j^{\prime}-j\right),
\end{gathered}
$$

the expressions of the elements of the $2 \times 2$ blocks in Table II are simplified.

If the intra-atomic ferromagnetic exchange integral $K$ is large as compared to the interatomic exchange $-K \gg|j|$, $\left|j^{\prime}\right|,\left|j^{\prime \prime}\right|$ — the energies of the lowest $S$ singlet and $T$ triplet eigenstates of the $2 \times 2$ blocks in Table II are

$$
\begin{aligned}
E_{S} & =3 A-\frac{3 B^{2}}{4 K-2 A} \\
& =\frac{3}{4}\left(j+j^{\prime}+2 j^{\prime \prime}\right)-\frac{3\left(j+j^{\prime}-2 j^{\prime \prime}\right)^{2}}{64 K-8\left(j+j^{\prime}+2 j^{\prime \prime}\right)},
\end{aligned}
$$

Notice that the largest components $S_{1}$ and $T_{1}$ on the lowest $S$ singlet and $T$ triplet, as well as $Q$, span the model space for the $S=1$ model Hamiltonian discussed in the following section.

\section{B. The spin Hamiltonian in the $S=1$ model space}

This space generates one singlet, one triplet, and one quintet states, and the corresponding wave functions are entirely determined by the total spin angular momentum. Since by hypothesis $S=1$, the basis set for this model Hamiltonian corresponds to the first three elements of Eq. (28). Hence,

$$
\begin{gathered}
S=\frac{1}{\sqrt{3}}\left\{T_{A}^{+} T_{B}^{-}+T_{A}^{-} T_{B}^{+}-T_{A}^{0} T_{B}^{0}\right\}, \\
T=\frac{1}{\sqrt{2}}\left\{T_{A}^{+} T_{B}^{-}-T_{A}^{-} T_{B}^{+}\right\}, \\
Q=\frac{1}{\sqrt{6}}\left\{T_{A}^{+} T_{B}^{-}+T_{A}^{-} T_{B}^{+}+2 T_{A}^{0} T_{B}^{0}\right\},
\end{gathered}
$$

and, hence, the only degrees of freedom concern the two energy differences between these three states. ${ }^{26,31}$ Consequently, the effective Hamiltonian involves only two parameters. Let us express the spectrum by

$$
\begin{aligned}
& E_{T}-E_{Q}=2 J_{2}, \\
& E_{S}-E_{Q}=3 J_{1} .
\end{aligned}
$$

Taking again the energy of the quintet state as the zero, the spectral decomposition is

$$
\hat{H}^{\mathrm{eff}}=3 J_{1}|S\rangle\left\langle S\left|+2 J_{2}\right| T\right\rangle\langle T|+0| Q\rangle\langle Q|
$$

and the matrix representation of the effective Hamiltonian in the basis $\left\{D_{i}^{\prime}\right\}$ defined in Eq. (28) can be calculated and takes the form given in Table III. This effective Hamiltonian can be specified for the $M_{S}=1$ subspace, which contains the 
TABLE III. Matrix representation of model Hamiltonian in the $S=1$ model space in the $D_{1}^{\prime}, D_{2}^{\prime}, D_{3}^{\prime}$ basis set; Eq. (28).

\begin{tabular}{cccc}
\hline \hline$\hat{H}^{\mathrm{eff}}$ & $D_{1}^{\prime}$ & $D_{2}^{\prime}$ & $D_{3}^{\prime}$ \\
\hline$D_{1}^{\prime}$ & $J_{1}+J_{2}$ & & \\
$D_{2}^{\prime}$ & $J_{1}-J_{2}$ & $J_{1}+J_{2}$ & \\
$D_{3}^{\prime}$ & $-J_{1}$ & $-J_{1}$ & $J_{1}$ \\
\hline \hline
\end{tabular}

$M_{S}=1$ triplet and quintet states. The degeneracies between $M_{S}=0$ and $M_{S}=1$ eigenvalues impose the Hamiltonian expression in Table IV.

Notice that the usual HDVV Hamiltonian takes the form

$$
\hat{H}^{\mathrm{HDVV}}=-\sum_{\langle i, j\rangle} J\left(\hat{S}_{i} \cdot \hat{S}_{j}-\hat{1}\right),
$$

when the quintet energy is taken as the origin, and, consequently, it only introduces one parameter and results in a spectrum where the energy differences are $E_{T}-E_{Q}=2 J$ and $E_{S}-E_{Q}=3 J$. The exact spectrum of a system with two magnetic centers with $S=1$ has no reason to strictly obey this $\frac{2}{3}$ ratio, and in order to take into account any possible deviation the biquadratic terms are to be considered. Modifying Eq. (6) to have the same energy zero,

$$
\hat{H}^{\mathrm{HDVV}}=-\sum_{\langle i, j\rangle} J\left[\hat{S}_{i} \cdot \hat{S}_{j}+\lambda\left(\hat{S}_{i} \cdot \hat{S}_{j}\right)^{2}-(1+\lambda) \hat{1}\right],
$$

and identifying Eqs. (34) and (36), we have

$$
\begin{gathered}
J=J_{2}, \\
\lambda=\left(1-J_{1} / J_{2}\right) .
\end{gathered}
$$

It is also interesting to relate $J_{1}$ and $J_{2}$ (or $J$ and $\lambda$ ) appearing in the $S=1$ HDVV Hamiltonian to the integrals appearing in the $S=\frac{1}{2}$ HDVV Hamiltonian. To the second order

$$
\begin{gathered}
J_{1}=\frac{1}{4}\left(j+j^{\prime}+2 j^{\prime \prime}\right)-\frac{\left(j+j^{\prime}-2 j^{\prime \prime}\right)^{2}}{64 K-8\left(j+j^{\prime}+2 j^{\prime \prime}\right)}, \\
J=J_{2}=\frac{1}{4}\left(j+j^{\prime}+2 j^{\prime \prime}\right)-h-\frac{\left(j^{\prime}-j\right)^{2}}{8\left(2 K-j^{\prime \prime}+2 h-2 h^{\prime}\right)} .
\end{gathered}
$$

Keeping the leading term $K$ in the second-order energy denominators, the amplitude $\lambda$ of the biquadratic term can be expressed as

$\lambda=\frac{J_{2}-J_{1}}{J_{2}}=\frac{1}{J_{2}}\left[-h+\frac{\left(3 j-j^{\prime}-2 j^{\prime \prime}\right)\left(3 j^{\prime}-j-2 j^{\prime \prime}\right)}{64 K}\right]$.

Hence, the biquadratic term $\lambda$ has a double origin; first the existence of a four-body contribution $h$ and, second, the coupling between the three-dimensional model space spanned by the product of atomic triplet states with the states that involve the atomic neutral singlet states.
TABLE IV. Matrix representation of the model Hamiltonian in the $S=1$ model space in the $M_{S}=1$ subspace.

\begin{tabular}{ccc}
\hline \hline$\hat{H}^{\text {eff }}$ & $T_{A}^{0} T_{B}^{+}$ & $T_{A}^{+} T_{B}^{0}$ \\
\hline$T_{A}^{0} T_{B}^{+}$ & $J_{2}$ & \\
$T_{A}^{+} T_{B}^{0}$ & $-J_{2}$ & $J_{2}$ \\
\hline \hline
\end{tabular}

\section{A PERTURBATIVE EXPANSION OF THE HDVV HAMILTONIAN FROM A HUBBARD-TYPE HAMILTONIAN}

Starting from a simple valence bond Hamiltonian, analogous to the Hubbard Hamiltonian for systems with one orbital per site, it is possible to obtain a perturbative analytic derivation of the two different spin Hamiltonians described in the preceding section. The bielectronic model Hamiltonian (i) introduces $t=t_{a b}$ and $t^{\prime}=t_{a^{\prime} b^{\prime}}$ hopping integrals between the $a$ and $b$ and $a^{\prime}$ and $b^{\prime}$ orbitals, respectively, which couple the neutral and singly ionic VB determinants with the doubly ionic ones; (ii) attributes an energy $U$ and $2 U$ to the singly and doubly ionic determinants, respectively, and; (iii) introduces the previously discussed atomic spectroscopy in the neutral determinants subset.

The resulting Hamiltonian matrix is given in Table V, where $I_{11}$ to $I_{18}$ are the singly ionic determinants:

$$
\begin{aligned}
& I_{11}=\operatorname{det}\left(a \bar{a} a^{\prime} \bar{b}^{\prime}\right), \\
& I_{12}=\operatorname{det}\left(a \bar{a} \bar{a}^{\prime} b^{\prime}\right), \\
& I_{13}=\operatorname{det}\left(a a^{\prime} \bar{a}^{\prime} \bar{b}\right), \\
& I_{14}=\operatorname{det}\left(\bar{a} a^{\prime} \bar{a}^{\prime} b\right), \\
& I_{15}=\operatorname{det}\left(a^{\prime} \bar{b}^{\prime} \bar{b} b\right), \\
& I_{16}=\operatorname{det}\left(\bar{a}^{\prime} b^{\prime} \bar{b} b\right), \\
& I_{17}=\operatorname{det}\left(a b^{\prime} \bar{b} \bar{b}^{\prime}\right), \\
& I_{18}=\operatorname{det}\left(\bar{a} b \bar{b}^{\prime} b^{\prime}\right) .
\end{aligned}
$$

The doubly ionic determinants only interact with singly ionic ones through $t$ parameters. Their contribution to the $S=\frac{1}{2}$ and $S=1$ spin Hamiltonians appears at the fourth order of perturbation as $t^{4} / U^{3}$ terms. These contributions may be neglected as compared to the leading fourth-order corrections that are proportional to $t^{4} / K U^{2}$ as will be analyzed below.

\section{A. Derivation of the $S=\frac{1}{2}$ spin Hamiltonian}

Applying the QDPT using the six-dimensional model space, $D_{1}$ to $D_{6}$, as the model space, one finds the following order-by-order contributions. To the first order, the integrals $j$ reduce to the direct exchange integrals: $j^{(1)}=2 K_{a b}, j^{\prime(1)}$ $=2 K_{a^{\prime} b^{\prime}}$, and $j^{\prime \prime(1)}=2 K_{a b^{\prime}}=2 K_{a^{\prime} b}$, whereas the four-body integrals are zero; $h^{(1)}=h^{\prime(1)}=h^{\prime \prime(1)}=0 \quad\left(\right.$ where $j^{(i)}, j^{\prime(i)}$, $j^{\prime \prime(i)}, h^{(i)}, h^{\prime(i)}, h^{\prime(i)}$ include all order corrections of the perturbation theory up to $i$ ). The second-order correction does not affect the four-electron integrals but introduces the kinetic exchange terms in the $J$ integrals: 
TABLE V. Matrix representation of the Hubbard-type model Hamiltonian projected onto the basis of the neutral determinants $D_{1}$ to $D_{10}$, and the relevant singly ionic determinants $I_{11}$ to $I_{18}$.

\begin{tabular}{cccccccccccccccccccc}
\hline \hline$D_{1}$ & 0 & & & & & & & & & & & & & & & & \\
$D_{2}$ & 0 & 0 & & & & & & & & & & & & & & \\
$D_{3}$ & 0 & 0 & $2 K$ & & & & & & & & & & & & & \\
$D_{4}$ & 0 & 0 & 0 & $2 K$ & & & & & & & & & & & & \\
$D_{5}$ & 0 & 0 & $-K$ & $-K$ & $2 K$ & & & & & & & & & & & \\
$D_{6}$ & 0 & 0 & $-K$ & $-K$ & 0 & $2 K$ & & & & & & & & & & \\
$D_{7}$ & 0 & 0 & 0 & 0 & 0 & 0 & $6 K$ & & & & & & & & & \\
$D_{8}$ & 0 & 0 & 0 & 0 & 0 & 0 & 0 & $6 K$ & & & & & & & & \\
$D_{9}$ & 0 & 0 & 0 & 0 & 0 & 0 & $K$ & $K$ & $6 K$ & & & & & & & \\
$D_{10}$ & 0 & 0 & 0 & 0 & 0 & 0 & $K$ & $K$ & 0 & $6 K$ & & & & & & \\
$I_{11}$ & $-t$ & 0 & 0 & $t$ & 0 & 0 & $t^{\prime}$ & 0 & 0 & 0 & $U$ & & & & & \\
$I_{12}$ & 0 & $t$ & $-t$ & 0 & 0 & 0 & $-t^{\prime}$ & 0 & 0 & 0 & 0 & $U$ & & & & \\
$I_{13}$ & $-t^{\prime}$ & 0 & $t^{\prime}$ & 0 & 0 & 0 & 0 & $t$ & 0 & 0 & 0 & 0 & $U$ & & & \\
$I_{14}$ & 0 & $t^{\prime}$ & 0 & $-t^{\prime}$ & 0 & 0 & 0 & $-t$ & 0 & 0 & 0 & 0 & 0 & $U$ & & & \\
$I_{15}$ & $t$ & 0 & 0 & $-t$ & 0 & 0 & 0 & $-t^{\prime}$ & 0 & 0 & 0 & 0 & 0 & 0 & $U$ & & \\
$I_{16}$ & 0 & $-t$ & $t$ & 0 & 0 & 0 & 0 & $t^{\prime}$ & 0 & 0 & 0 & 0 & 0 & 0 & 0 & $U$ & \\
$I_{17}$ & $t^{\prime}$ & 0 & $-t^{\prime}$ & 0 & 0 & 0 & $-t$ & 0 & 0 & 0 & 0 & 0 & 0 & 0 & 0 & 0 & $U$ \\
$I_{18}$ & 0 & $-t^{\prime}$ & 0 & $t^{\prime}$ & 0 & 0 & $t$ & 0 & 0 & 0 & 0 & 0 & 0 & 0 & 0 & 0 & 0 & $U$ \\
\hline \hline
\end{tabular}

$$
\begin{gathered}
j^{(2)}=2 K_{a b}-\frac{4 t^{2}}{U}, \\
j^{(2)}=2 K_{a^{\prime} b^{\prime}}-\frac{4 t^{\prime 2}}{U}, \\
j^{\prime \prime(2)}=2 K_{a^{\prime} b} \text { if } t_{a b^{\prime}}=t_{a^{\prime} b}=0 .
\end{gathered}
$$

The third order only affects the $j^{\prime \prime}$ integrals as

$$
j^{\prime \prime(3)}=2 K_{a^{\prime} b}-4 K \frac{t^{2}+t^{\prime 2}}{U^{2}} .
$$

The fourth-order corrections on the integrals are numerous and it is convenient to concentrate on their origin. The amplitude $h$ associated with the four-body operator, $h=\left|D_{2}\right\rangle\left\langle D_{1}\right|$, comes from fourth-order processes of the type

$$
h=\sum_{i=11,18} \sum_{j=11,18} \sum_{k=7,8} \frac{\left\langle D_{2}|\hat{V}| I_{i}\right\rangle\left\langle I_{i}|\hat{V}| D_{k}\right\rangle\left\langle D_{k}|\hat{V}| I_{j}\right\rangle\left\langle I_{j}|\hat{V}| D_{1}\right\rangle}{6 K U^{2}}
$$

and similarly for $h^{\prime}$. From Table V, it may be verified that these four-body operators behave as $t^{2} t^{\prime 2} / U^{2} K$.

The fourth-order corrections are null for $j^{\prime \prime}$ and $h^{\prime \prime}$ since $D_{5}$ and $D_{6}$ are not coupled with the singly ionic determinants. The nonzero contributions to other integrals lead to the fourth-order corrected expressions

$$
\begin{gathered}
j^{(4)}=2 K_{a b}-\frac{4 t^{\prime 2}}{U}-\frac{8 t^{2} t^{\prime 2}}{3 K U^{2}}, \\
j^{\prime(4)}=2 K_{a^{\prime} b^{\prime}}-\frac{4 t^{\prime 2}}{U}-\frac{8 t^{2} t^{\prime 2}}{3 K U^{2}}, \\
h^{(4)}=-\frac{4 t^{2} t^{\prime 2}}{3 K U^{2}}=h^{\prime(4)}
\end{gathered}
$$

Consequently, at this level, four-electron terms appear that can be of importance in systems where $t^{2} t^{\prime 2} / K U^{2}$ is not negligible as compared to the second-order contributions, which are $j \approx-4 t^{2} / U$ and $j^{\prime} \approx-4 t^{\prime 2} / U$. This requires $8 t^{4} / 3 K U^{2} \approx 4 t^{2} / U$, or $K \approx 2 t^{2} / 3 U \approx j / 6$. Hence, the fourelectron terms must be introduced when the intersite couplings are not negligible as compared to the intrasite direct exchange.

\section{B. Derivation of the $S=1$ spin Hamiltonian}

The matrix representation of the Hubbard-type Hamiltonian reported on Table $\mathrm{V}$ can be transformed to the basis of the eigenfunctions of the HDVV Hamiltonian given in Eq. (29). The result of such a transformation is given in Table VI, where $F_{1}$ to $F_{4}$, defined as 
TABLE VI. Matrix representation of the Hubbard-type model Hamiltonian projected onto the basis of the $Q, T_{1}, T_{2}, T_{3}, S_{1}, S_{2}$ states, Eq. (29), the $F_{1}$ to $F_{4}$ states combining $D_{7}$ to $D_{10}$, cf. Eq. (46) and the relevant singly ionic determinants $I_{11}$ to $I_{18}$. For convenience the coefficients $a, b$, and $c$ are used instead of $(\sqrt{2} / 2)$, $(\sqrt{3} / 2)$, and $\frac{1}{2}$, respectively.

\begin{tabular}{|c|c|c|c|c|c|c|c|c|c|c|c|c|c|c|c|c|c|c|}
\hline$Q$ & 0 & & & & & & & & & & & & & & & & & \\
\hline$T_{1}$ & 0 & 0 & & & & & & & & & & & & & & & & \\
\hline$S_{1}$ & 0 & 0 & 0 & & & & & & & & & & & & & & & \\
\hline$T_{2}$ & 0 & 0 & 0 & $2 K$ & & & & & & & & & & & & & & \\
\hline$T_{3}$ & 0 & 0 & 0 & 0 & $2 K$ & & & & & & & & & & & & & \\
\hline$S_{2}$ & 0 & 0 & 0 & 0 & 0 & $4 K$ & & & & & & & & & & & & \\
\hline$F_{1}$ & 0 & 0 & 0 & 0 & 0 & 0 & $4 K$ & & & & & & & & & & & \\
\hline$F_{2}$ & 0 & 0 & 0 & 0 & 0 & 0 & 0 & $6 K$ & & & & & & & & & & \\
\hline$F_{3}$ & 0 & 0 & 0 & 0 & 0 & 0 & 0 & 0 & $6 K$ & & & & & & & & & \\
\hline$F_{4}$ & 0 & 0 & 0 & 0 & 0 & 0 & 0 & 0 & 0 & $8 K$ & & & & & & & & \\
\hline$I_{11}$ & 0 & $-a t$ & $-b t$ & $a t$ & 0 & $-c t$ & $c t^{\prime}$ & $a t^{\prime}$ & 0 & $c t^{\prime}$ & $U$ & & & & & & & \\
\hline$I_{12}$ & 0 & $-a t$ & $b t$ & at & 0 & $c t$ & $-c t^{\prime}$ & $-a t^{\prime}$ & 0 & $-c t^{\prime}$ & 0 & $U$ & & & & & & \\
\hline$I_{13}$ & 0 & $-a t^{\prime}$ & $-b t^{\prime}$ & $-a t^{\prime}$ & 0 & $-c t^{\prime}$ & $c t$ & $-a t$ & 0 & $c t$ & 0 & 0 & $U$ & & & & & \\
\hline$I_{14}$ & 0 & $-a t^{\prime}$ & $b t^{\prime}$ & $-a t^{\prime}$ & 0 & $c t^{\prime}$ & $-c t$ & $a t$ & 0 & $-c t$ & 0 & 0 & 0 & $U$ & & & & \\
\hline$I_{15}$ & 0 & at & $b t$ & $-a t$ & 0 & $c t$ & $-c t^{\prime}$ & $a t^{\prime}$ & 0 & $-c t^{\prime}$ & 0 & 0 & 0 & 0 & $U$ & & & \\
\hline$I_{16}$ & 0 & $a t$ & $-b t$ & $-a t$ & 0 & $-c t$ & $c t^{\prime}$ & $-a t^{\prime}$ & 0 & $c t^{\prime}$ & 0 & 0 & 0 & 0 & 0 & $U$ & & \\
\hline$I_{17}$ & 0 & $a t^{\prime}$ & $b t^{\prime}$ & $a t^{\prime}$ & 0 & $c t^{\prime}$ & $-c t$ & $-a t$ & 0 & $-c t$ & 0 & 0 & 0 & 0 & 0 & 0 & $U$ & \\
\hline$I_{18}$ & 0 & $a t^{\prime}$ & $-b t^{\prime}$ & $a t^{\prime}$ & 0 & $-c t^{\prime}$ & $c t$ & $a t$ & 0 & $c t$ & 0 & 0 & 0 & 0 & 0 & 0 & 0 & $U$ \\
\hline
\end{tabular}

$$
\begin{gathered}
F_{1}=\frac{1}{2}\left\{D_{7}+D_{8}-D_{9}-D_{10}\right\}, \\
F_{2}=\frac{1}{\sqrt{2}}\left\{D_{7}-D_{8}\right\}, \\
F_{3}=\frac{1}{\sqrt{2}}\left\{D_{9}-D_{10}\right\}, \\
F_{4}=\frac{1}{2}\left\{D_{7}+D_{8}+D_{9}+D_{10}\right\},
\end{gathered}
$$

diagonalize the Hamiltonian in this subspace and account for the strong $K$ coupling existing between $D_{7}$ and $D_{8}$ with $D_{9}$ and $D_{10}$.

At the second order of the QDPT, the energy levels are

$$
\begin{gathered}
E_{Q}^{(2)}=0, \\
E_{T}^{(2)}=-2 \frac{t^{2}+t^{\prime 2}}{U}, \\
E_{S}^{(2)}=-3 \frac{t^{2}+t^{\prime 2}}{U} .
\end{gathered}
$$

This is

$$
J_{1}^{(2)}=J_{2}^{(2)}=-\frac{t^{2}+t^{\prime 2}}{U}
$$

and the four-body contribution $[\lambda$ in Eq. (6) and Eq. (40)] is zero.

Up to the fourth order of the QDPT it can be sown that

$$
E_{Q}^{(4)}=0
$$

$$
\begin{gathered}
E_{T}^{(4)}=-2 \frac{t^{2}+t^{\prime 2}}{U}-2 \frac{\left(t^{2}-t^{\prime 2}\right)^{2}}{K U^{2}}, \\
E_{S}^{(4)}=-3 \frac{t^{2}+t^{\prime 2}}{U}-3 \frac{\left(t^{2}-t^{\prime 2}\right)^{2}}{K U^{2}}+\frac{9}{4} \frac{\left(t^{2}-t^{\prime 2}\right)^{2}}{K U^{2}}-\frac{15}{2} \frac{t^{2} t^{\prime 2}}{K U^{2}}
\end{gathered}
$$

and, consequently,

$$
\begin{gathered}
J_{2}^{(4)}=-\frac{t^{2}+t^{\prime 2}}{U}-\frac{\left(t^{2}-t^{\prime 2}\right)^{2}}{K U^{2}}, \\
J_{1}^{(4)}=J_{2}^{(4)}+\frac{3}{4} \frac{\left(t^{2}-t^{\prime 2}\right)^{2}}{K U^{2}}-\frac{5}{2} \frac{t^{2} t^{\prime 2}}{K U^{2}} .
\end{gathered}
$$

The last two terms of Eq. (49) govern the deviation from a pure HDVV Hamiltonian based on the $S=1$ local triplet state that predicts that $J=J_{1}=J_{2}$. The deviation becomes important when $t^{4} / K U^{2}$ and/or $t^{\prime 4} / K U^{2}$ is not negligible in front of $K$. In the case of the acetylene molecule discussed in Sec. VII, $t=t^{\prime}$, and, hence, the deviation is $J_{1}^{(4)}=J_{2}^{(4)}$ $-\frac{5}{2} t^{4} / K U^{2}$.

\section{NUMERICAL EXTRACTION OF SPIN HAMILTONIANS FROM ACCURATE CONFIGURATION-INTERACTION CALCULATIONS}

This section reports numerical applications of the formalism presented above to two cases, the $\mathrm{Ni}_{2} \mathrm{~F}_{11}$ cluster model of the $\mathrm{K}_{2} \mathrm{NiF}_{4}$ perovskite and the $\pi$ system of the acetylene molecule. 


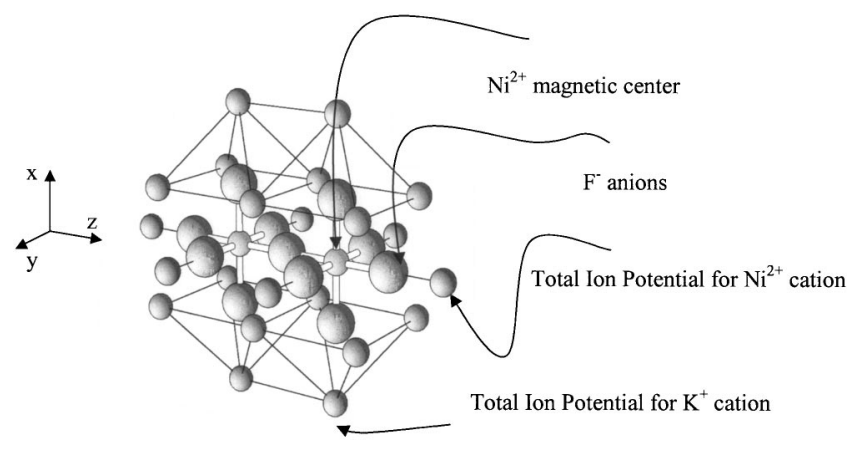

FIG. 2. The embedded $\mathrm{Ni}_{2} \mathrm{~F}_{11}$ cluster model representation of $\mathrm{K}_{2} \mathrm{NiF}_{4}$.

\section{A. Ab initio model Hamiltonian for $\mathrm{K}_{2} \mathrm{NiF}_{4}$}

The $\mathrm{K}_{2} \mathrm{NiF}_{4}$ perovskite has been represented by an embedded-cluster model, which is schematically shown in Fig. 2. This cluster can be described as $\left(\mathrm{Ni}_{2} \mathrm{~F}_{11}\right)\left(\mathrm{K}_{16} \mathrm{Ni}_{6}\right)$ [point charges (PC's)] and may be seen as containing three different regions, the first is $\left(\mathrm{Ni}_{2} \mathrm{~F}_{11}\right)$ and contains the atoms that are explicitly quantum mechanically treated, the second region provides an adequate quantum embedding to the first region and includes the $\left(\mathrm{K}_{8} \mathrm{Ni}_{6}\right)$ moiety, which in turn is described by total ion potentials, ${ }^{44}$ TIP's, representing the $\mathrm{Ni}^{2+}$ and $\mathrm{K}^{+}$cations surrounding the $\mathrm{F}^{-}$anions. The role of the TIP's is to avoid an excessive polarization of the anions towards the PC's that surround the first and second region. ${ }^{45}$ The point charges provide the long-range Madelung potential; fractional charges obtained by means of Evjen's method ${ }^{46}$ are used in the cluster edge to provide an accurate potential in the inner region. The $1 s^{2}$ electrons of the $\mathrm{F}^{-}$ anions and the $1 s^{2} 2 s^{2} 3 p^{6} 3 s^{2} 3 p^{6}$ electrons of $\mathrm{Ni}^{2+}$ have been included in a pseudopotential ${ }^{47}$ and the total number of electrons included in the calculations corresponds to an ionic description, although the total charge of the resulting embedded-cluster model is zero. The $\mathrm{Ni}^{2+}$ basis set is of
$(3 s 3 p 6 d)$ quality and is contracted to $[2 s 2 p 3 d]$. For the fluorine bridging anion, the basis set is of triple- $z$ plus polarization quality whereas a double- $z$ basis has been used for the external fluorine anions. The overall basis set is similar to that used in a previous work ${ }^{31}$ and the difference in the calculated magnetic coupling constant is almost negligible $(<4$ $\mathrm{K}$ or $0.35 \mathrm{meV}$ ).

The molecular orbitals are obtained from a restricted open-shell Hartree-Fock calculation in the quintet state, and the unpaired electrons occupy the two linear combinations of the $3 d_{x^{2}-y^{2}}$ and the $3 d_{z^{2}}$ (see Fig. 2) magnetic orbitals centered on the $\mathrm{Ni}$ atoms with variational delocalization tails in the neighboring fluorine atoms. The complete active space (CAS) involves four electrons in four orbitals and may be regarded as a full valence bond space on which it is easy to identify the six neutral VB determinants. However, the configuration interaction (CI) calculations performed according to the difference dedicated CI procedure ${ }^{48,49}$ involve double excitations from the closed-shell electrons to the active space, double excitations from the active space electrons to the virtual space and all the possible single excitations on top of all CAS determinants. The CI expansion runs over more than $10^{5}$ determinants. In this CI space; one has to identify the six eigenvectors with largest neutral valence bond character, i.e., the largest projection onto the model space. Due to the existence of low-lying ligand to metal charge-transfer states, the six roots of interest are not the six lowest eigenstates. They have the ranks 1, 2, and 3 (those corresponding to the local triplets as expected), and 6, 7, and 13. Finding the higher roots require the use of the recently proposed Lagrange-Newton-Raphson-diagonalization (LNRd) technique. ${ }^{50-52}$ The corresponding energies and the projection of the eigenvectors in the basis $D_{1}$ to $D_{6}$ are given in Table VII. These projections happen to be orthogonal and have been renormalized. As expected, in the ground-state wave function, the coefficients of the determinants $D_{3}$ and $D_{4}$, which are coupled with the ionic VB determinants, i.e., give rise to electronic delocalization, are slightly larger

TABLE VII. For the $\mathrm{Ni}_{2} \mathrm{~F}_{11}$ embedded cluster model of the $\mathrm{K}_{2} \mathrm{NiF}_{4}$ perovskite, the eigenvalues (in meV) of the six electronic eigenstates with the largest projection in the valence-bond model space spanned by $D_{1}$ to $D_{6}$ determinants [Eq. (29)] relative to the quintet ground state and their projection onto the model space. In parentheses, the expected coefficients of an exact HDVV Hamiltonian.

\begin{tabular}{ccccccc}
\hline \hline $\begin{array}{c}\text { States } \\
\text { Energies (meV) }\end{array}$ & $S_{1}$ & $T_{1}$ & $Q$ & $T_{2}$ & $T_{3}$ & $S_{2}$ \\
\hline$D_{1}$ & 0.5773 & 0.7070 & 0.4082 & -0.0058 & 0.0000 & -0.0013 \\
& $(0.5774)$ & $(0.7071)$ & $(0.4082)$ & $(0.0000)$ & $(0.0000)$ & $(0.0000)$ \\
$D_{2}$ & 0.5773 & -0.7070 & 0.4082 & 0.0058 & 0.0000 & -0.0013 \\
& $(0.5774)$ & $(-0.7071)$ & $(0.4082)$ & $(0.0000)$ & $(0.0000)$ & $(0.0000)$ \\
$D_{3}$ & -0.2898 & -0.0058 & 0.4082 & -0.7070 & 0.0000 & -0.4993 \\
& $(-0.2887)$ & $(0.0000)$ & $(0.4082)$ & $(-0.7071)$ & $(0.0000)$ & $(-0.5000)$ \\
$D_{4}$ & -0.2898 & 0.0058 & 0.4082 & 0.7070 & 0.0000 & -0.4993 \\
& $(-0.2887)$ & $(0.0000)$ & $(0.4082)$ & $(0.7071)$ & $(0.0000)$ & $(-0.5000)$ \\
$D_{5}$ & -0.2875 & 0.0000 & 0.4082 & 0.0000 & 0.7071 & 0.5006 \\
& $(-0.2887)$ & $(0.0000)$ & $(0.4082)$ & $(0.0000)$ & $(0.7071)$ & $(0.5000)$ \\
$D_{6}$ & -0.2875 & 0.0000 & 0.4082 & 0.0000 & -0.7071 & 0.5006 \\
& $(-0.2887)$ & $(0.0000)$ & $(0.4082)$ & $(0.0000)$ & $(-0.7071)$ & $(0.5000)$ \\
\hline \hline
\end{tabular}


than those of the determinants $D_{5}$ and $D_{6}$. For an exact $S$ $=1 \mathrm{HDVV}$ Hamiltonian, the composition of the six eigenstates would be somewhat different as shown in parentheses in the table. The small deviations are responsible for the appearance of biquadratic forms in the spin Hamiltonian. Using the spectral definition of the effective Hamiltonian permits to obtain the values of the seven elements defining the matrix representation of the effective Hamiltonian given in Table II.

The values found are

$$
\begin{gathered}
j=8.57 \mathrm{meV}, \\
j^{\prime}=-29.18 \mathrm{meV}, \\
j^{\prime \prime}=1.69 \mathrm{meV}, \\
K=1151.34 \mathrm{meV}, \\
h=0.00 \mathrm{meV}, \\
h^{\prime}=-0.20 \mathrm{meV}, \\
h^{\prime \prime}=-0.25 \mathrm{meV} .
\end{gathered}
$$

These results call for the following comments.

(i) The intra-atomic effective exchange integral $K$ is 40 times larger than the largest effective interatomic exchange integral. This is a case where the $S=1$ pure HDVV Hamiltonian, without the quadratic term, should be appropriate, and actually

$$
\frac{E_{T}-E_{Q}}{E_{S}-E_{Q}}=\frac{8.77}{12.95}=0.677,
$$

slightly larger than the $\frac{2}{3}$ ratio predicted by this Hamiltonian.

(ii) The largest antiferromagnetic interatomic exchange $j^{\prime}$ concerns the $3 d_{z^{2}}$ orbitals, which have the largest differential overlap directed along the $180^{\circ}$ magnetic path.

(iii) The effective coupling $j$ between the $3 d_{x^{2}-y^{2}}$ orbitals remains positive, due to the first-order ferromagnetic exchange which prevails over the delocalization or over the spin polarization effects.

(iv) The integral $j^{\prime \prime}$ between the $3 d_{x^{2}-y^{2}}$ on one center and the $3 d_{z^{2}}$ on the other center is non-negligible. It is positive, the contribution of the antiferromagnetic Anderson mechanism between these orbitals being zero.

(v) The four-body operators $h, h^{\prime}$, and $h^{\prime \prime}$ are weak but nonzero. They do not obey the relations $h^{\prime}=h$ and $h^{\prime \prime}=0$ obtained by permuting the Hubbard Hamiltonian, which appears to be oversimplified.

From the eigenenergies in Table VII, it is also easy to establish the $S=1$ HDVV Hamiltonian since $J_{2}=J=-4.39$ $\mathrm{meV}, J_{1}=-4.32 \mathrm{meV}$, and $\lambda=1-J_{1} / J_{2}=0.07 / 4.39=0.016$, confirming the weakness of the biquadratic terms in this system.

The role of the coupling between the three neutral states generated by the local atomic triplets and the neutral states involving the local atomic singlets is also shown. If this coupling is not taken into account, the energy of the $T$ triplet state is $\left(j+j^{\prime}+2 j^{\prime \prime}\right) / 2=-8.62 \mathrm{meV}$. The perturbation stabilizes this triplet state by $-\left(j-j^{\prime}\right)^{2} / 8 K=-0.15 \mathrm{meV}$, leading to the final value of $-8.77 \mathrm{meV}$. The stabilization of the singlet state is $3\left(j+j^{\prime}-2 j^{\prime \prime}\right)^{2} / 64 K=0.02 \mathrm{meV}$, much smaller than that of the triplet state.

\section{B. Ab initio model Hamiltonian for the $\pi$ system of the acetylene molecule}

The $S=\frac{1}{2}$ HDVV Hamiltonian has been proved to be a very efficient tool for the study of conjugate hydrocarbons, where each carbon atom brings one $\pi$ electron in a $2 p_{z}(\pi)$ atomic orbital. ${ }^{7,8}$ This success is somewhat unexpected, since these systems are considered to be highly delocalized (with a $|t| / U$ ratio close to 1$)$, but it can be rationalized in a similar way to that described in the preceding section. The effective antiferromagnetic coupling $J$ should not be identified with its second-order amplitude $J^{(2)}=-4 t^{2} / U$ but with the exact excitation energy of the Hubbard Hamiltonian for the dimer

$$
J=\frac{U-\sqrt{U^{2}+16 t^{2}}}{2} .
$$

When $J$ is extracted from the simplest conjugated molecule, namely, ethyne, for a set of C-C distances, one obtains an $r$-dependent HDVV Hamiltonian, which, properly complemented by a $\sigma$-bond force field, becomes a quantitative tool for the study of ground and excited states and can be directly applied to photochemistry. ${ }^{53}$ One may wonder whether such a model might be used for the treatment of the lowest states of poly-ynes; i.e., of $-\mathrm{C} \equiv \mathrm{C}-$ triple bonds, and considering either an $S=\frac{1}{2}$ or $S=1$ spin model Hamiltonian.

Accurate $a b$ initio $\mathrm{CI}$ calculations of the spectrum of the acetylene molecule, in a double-zeta plus polarization (DZP) basis set, have already been reported in the past, ${ }^{54,55}$ concentrating especially on the possible generation of a $S=\frac{1}{2}$ spin Hamiltonian. Table VIII reports the energy spectrum and the components of the relevant eigenvectors of a large CI expansion in the model space, for the equilibrium ground-state geometry. The $S_{1}$ and $S_{2}$ projections are strongly nonorthogonal. This is because there are two intruder singlet states in this section of the spectrum. To obtain a Hermitian operator, a further orthonormalization step is required and, rather than a $S^{-1 / 2}$ symmetric orthogonalization, a GrammSchmidt-type orthogonalization of $S_{2}$ to $S_{1}$ is preferable. ${ }^{54}$ The resulting matrix elements of the $S=\frac{1}{2}$ spin Hamiltonian are the following (in atomic units):

$$
\begin{gathered}
j=j^{\prime}=-0.196, \\
j^{\prime \prime}=-0.004, \\
K=0.032, \\
h=-0.003, \\
h^{\prime}=-0.005, \\
h^{\prime \prime}=-0.012 .
\end{gathered}
$$


TABLE VIII. Eigenvalues of the six electronic states of the acetylene molecule with the largest projection in the valence-bond model space spanned by $D_{1}$ to $D_{6}$ determinants relative to the quintet ground state and their projection in the model space.

\begin{tabular}{ccccccc}
\hline \hline $\begin{array}{c}\text { States } \\
\text { Energies (hartree) }\end{array}$ & $\begin{array}{c}S_{1} \\
-0.367\end{array}$ & $\begin{array}{c}T_{1} \\
-0.189\end{array}$ & $\begin{array}{c}T_{2} \\
-0.138\end{array}$ & $\begin{array}{c}Q \\
0.0\end{array}$ & $\begin{array}{c}S_{2} \\
0.064\end{array}$ & $\begin{array}{c}T_{3} \\
0.064\end{array}$ \\
\hline$D_{1}$ & 0.525 & 0.707 & 0.000 & 0.408 & 0.416 & 0.000 \\
$D_{2}$ & 0.525 & -0.707 & 0.000 & 0.408 & 0.416 & 0.000 \\
$D_{3}$ & -0.470 & 0.000 & 0.707 & 0.408 & 0.148 & 0.000 \\
$D_{4}$ & -0.470 & 0.000 & -0.707 & 0.408 & 0.148 & 0.000 \\
$D_{5}$ & -0.054 & 0.000 & 0.000 & 0.408 & 0.552 & 0.707 \\
$D_{6}$ & -0.054 & 0.000 & 0.000 & 0.408 & 0.552 & -0.707 \\
\hline \hline
\end{tabular}

From these parameters it appears that for this problem, the intra-atomic ferromagnetic exchange $K$ is smaller than the intersite antiferromagnetic exchange $j$. Moreover, some fourbody operators are large, $\sim 5 \%$ of $j$.

It is important to point out that one of the two abovecommented intruder states is of ionic character and lies 0.059 a.u. above the quintet state, while the other one appears at -0.083 a.u. below the quintet state, is of a neutral closedshell valence bond nature, and is spanned by the determinants $D_{7}$ and $D_{8}$. The coefficients of these determinants in the $S_{1}$ and $S_{2}$ singlet states are large and it is clear that they act as strong perturbers at the fourth order, resulting in strong deviations from a simple $S=\frac{1}{2}$ HDVV Hamiltonian.

From the energy differences

$$
\begin{aligned}
& E_{S}-E_{Q}=-0.367 \text { a.u., } \\
& E_{T}-E_{Q}=-0.189 \text { a.u., }
\end{aligned}
$$

the $S=1$ Hamiltonian is easily obtained, with $J_{1}=-0.123$ hartree and $J_{2}=-0.0945$ hartree or, equivalently, $J$ $=-0.0945$ hartree and $\lambda=-0.3016, \lambda$ being the amplitude of the four-body term appearing in Eq. (6). Notice that, in this case, the importance of the four-body operator is crucial in contrast to what has been found for the $\mathrm{K}_{2} \mathrm{NiF}_{4}$ compound. Such an extraction may be performed for several C-C interatomic distances and the resulting effective Hamiltonian may be interpolated in a polynomial form as a function of the $\mathrm{C}-\mathrm{C}$ distance.

The efficiency of the resulting spin Hamiltonian has been tested by computing the low-energy spectrum of the first poly-ynes, and comparing the vertical absorption and emission energies to those obtained using an $S=\frac{1}{2}$ spin Hamiltonian and reported in Ref. 55. The results in Table IX sug-

TABLE IX. Lowest singlet to triplet transition energies of even poly-ynes (in $\mathrm{eV}$ ). Results in parentheses refer to previous results using an $S=\frac{1}{2}$ spin Hamiltonian.

\begin{tabular}{lccc}
\hline \hline & $\mathrm{C}_{4} \mathrm{H}_{2}$ & $\mathrm{C}_{6} \mathrm{H}_{2}$ & $\mathrm{C}_{8} \mathrm{H}_{2}$ \\
\hline Vert. absorption & $3.62(3.79)^{\mathrm{a}}$ & $2.77(3.00)$ & $2.27(2.53)$ \\
Vert. emission & $2.43(2.53)$ & $1.77(1.90)$ & $1.37(1.50)$ \\
\hline \hline
\end{tabular}

a $a$ initio $\mathrm{Cl}$ calculations predict $3.84 \mathrm{eV}$. gest that although the $S=1$ spin Hamiltonian slightly underestimates the excitation energies, it gives rather reliable results.

\section{CONCLUSIONS}

The present work has analyzed the foundations and limits of $S=\frac{1}{2}$ and $S=1$ spin Hamiltonians for systems in which each site brings two unpaired electrons in two well-defined orbitals. The analysis is limited to dimers but it permits to reveal the underlying physics. From first-principles arguments it is shown that, beyond the usual $J_{i j}$ terms, the effective spin Hamiltonian (which for the dimer can always be defined from either a six-dimensional model space or a threedimensional space for $S=\frac{1}{2}$ and $S=1$, respectively) necessarily introduces four-body spin operators in the $S=\frac{1}{2}$ case and biquadratic terms in the $S=1$ formalism. The order of magnitude of these additional terms can be rationalized from a QDPT expansion starting from a Hubbard-type Hamiltonian. It is shown that both four-body and biquadratic terms behave as $\lambda t^{4} / K U^{2} \approx J^{2} / K$. Therefore, these terms play a negligible role when $K \gg|J|$, i.e., when the ferromagnetic intrasite direct exchange is much larger than the antiferromagnetic intersite kinetic exchange $\left(J \approx-4 t^{2} / U\right)$.

$A b$ initio accurate quantum chemical calculations, using extended basis sets and large configuration expansions, have been used to numerically derive effective spin Hamiltonians from the exact ones. In the case of $\mathrm{K}_{2} \mathrm{NiF}_{4}$ (or $\mathrm{KNiF}_{3}$ ), which involves $\mathrm{Ni}^{2+}$ with a very large intrasite direct exchange favoring the local fulfilling of Hund's rule, the fourbody operators and the biquadratic terms established from an analytical derivation remain with a very small amplitude $(3 \%$ of $J$ ). However, if one considers a carbon atom of a poly-yne as bringing also two unpaired electrons per site in two orthogonal $\pi$ bonds, the on-site ferromagnetic exchange and the intersite kinetic exchange are of the same order of magnitude. Consequently, the four-body operators in the $S=\frac{1}{2}$ and the biquadratic terms in the $S=1$ effective Hamiltonians become very large.

To summarize, the physical mechanisms governing the reduction from the all electron Hamiltonian to the spin-only Hamiltonians have been analyzed through the effective Hamiltonian theory. The conditions under which it is possible to reduce the full spin Hamiltonian to its simpler 
HDVV form have been specified. Ab initio calculations confirm the analytical derivation of these conditions.

\section{ACKNOWLEDGMENTS}

This research has been supported by the Spanish DGICYT (Grants Nos. PB98-1216-C02-01, PB98-1216-C02-01-
02, and PB98-1240-C02-01) and, in part, by Generalitat de Catalunya Grants Nos. 2001SGR-00043 and 2001SGR00315. The Laboratoire de Physique Quantique is Unité Mixte (UMR 5626) $d u$ CNRS. Bilateral exchange was supported by Grants Nos. HF2000-30 and PICS2001-13. I. de P.R.M. is grateful to the University of Barcelona for financial support.
${ }^{1}$ W. Heisenberg, Z. Phys. 49, 618 (1928).

${ }^{2}$ P. A. M. Dirac, The Principles of Quantum Mechanics, 3rd ed (Clarendon, Oxford, 1947). Chap. IX.

${ }^{3}$ C. Herring, in Magnetism, edited by G. T. Rado and H. Suhl (Academic, New York, 1965), Vol. 2B.

${ }^{4}$ B. H. Brandow, Rev. Mod. Phys. 39, 771 (1967).

${ }^{5}$ I. Lindgren and J. Morrison, Atomic Many Body Theory (Springer-Verlag, Berlin, 1982), p. 200.

${ }^{6} \mathrm{P}$. Durand and J. P. Malrieu, in Ab Initio Methods in Quantum Chemistry, edited by K. P. Lawley (Wiley, New York, 1987), Vol. 1, Chap. VI.

${ }^{7}$ J. P. Malrieu and D. Maynau, J. Am. Chem. Soc. 104, 3021 (1982).

${ }^{8}$ D. Maynau, M. Said, and J. P. Malrieu, J. Am. Chem. Soc. 105, 5244 (1983).

${ }^{9}$ R. K. Nesbet, Ann. Phys. 4, 87 (1958).

${ }^{10}$ R. K. Nesbet, Phys. Rev. 119, 658 (1960).

${ }^{11}$ R. K. Nesbet, Phys. Rev. 122, 1497 (1961).

${ }^{12}$ J. P. Malrieu and D. Maynau, J. Am. Chem. Soc. 104, 3021 (1982); D. Maynau and J. P. Malrieu, ibid. 104, 3029 (1982).

${ }^{13}$ A. H. McDonald, S. M. Girvin, and D. Yoshioka, Phys. Rev. B 37, 9753 (1988); 41, 2565 (1990).

${ }^{14}$ C. J. Calzado and J. P. Malrieu, Eur. Phys. J. B 21, 375 (2001).

${ }^{15}$ A. J. W. Wachters and W. C. Nieuwpoort, in Selected Topics in Molecular Physics, edited by E. Clementi (Verlag-Chemie, Weinheim, 1972), p. 135.

${ }^{16}$ G. Chen and W. A. Goddard III, Science 239, 899 (1988).

${ }^{17}$ R. L. Martin, J. Chem. Phys. 98, 8691 (1993).

${ }^{18}$ R. L. Martin, in Cluster Models for Surface and Bulk Phenomena, Vol. 283 of NATO Advanced Studies Institute, Series B: Physics, edited by G. Pacchioni, P. S. Bagus, and F. Parmigiani (Plenum New York, 1991), p. 485.

${ }^{19}$ M. S. Hybertsen, E. B. Stechel, M. Schluter, and D. R. Jennison, Phys. Rev. B 41, 11068 (1990).

${ }^{20}$ C. T. Chen, F. Sette, Y. Ma, M. S. Hybertsen, E. B. Stechel, W. M. C. Foulkes, M. Schluter, S. W. Cheong, A. S. Cooper, L. W. Rupp, B. Batlogg, Y. L. Soo, Z. H. Ming, A. Krol, and Y. H. Kao, Phys. Rev. Lett. 66, 104 (1991).

${ }^{21}$ M. S. Hybertsen, E. B. Stechel, W. M. C. Foulkes, and M. Schluter, Phys. Rev. B 45, 10032 (1992).

${ }^{22}$ W. E. Pickett, Rev. Mod. Phys. 61, 433 (1989).

${ }^{23}$ G. Y. Guo and W. M. Temmerman, J. Phys. C 21, L803 (1988).

${ }^{24}$ L. F. Mattheiss, Phys. Rev. B 49, 14050 (1994).

${ }^{25}$ R. V. Kasowski, M. H. Tsai, and J. D. Dow, Phys. Rev. B 41, 7744 (1990).

${ }^{26}$ F. Illas, J. Casanovas, M. A. Garcia-Bach, R. Caballol, and O. Castell, Phys. Rev. Lett. 71, 3549 (1993).
${ }^{27}$ I. de P. R. Moreira, F. Illas, C. J. Calzado, J. F. Sanz, J. P. Malrieu, N. Ben-Amor, and D. Maynau, Phys. Rev. B 59, R6593 (1999).

${ }^{28}$ J. Casanovas and F. Illas, J. Chem. Phys. 100, 8257 (1994).

${ }^{29}$ J. Casanovas, J. Rubio, and F. Illas, Phys. Rev. B 53, 945 (1996).

${ }^{30}$ C. de Graaf, F. Illas, R. Broer, and W. C. Nieuwpoort, J. Chem. Phys. 106, 3287 (1997).

${ }^{31}$ I. de P. R. Moreira and F. Illas, Phys. Rev. B 55, 4129 (1997).

${ }^{32}$ F. Illas, I. de P. R. Moreira, C. de Graaf, O. Castell, and J. Casanovas, Phys. Rev. B 56, 5069 (1997).

${ }^{33}$ I. de P. R. Moreira and F. Illas, Phys. Rev. B 60, 5179 (1999).

${ }^{34}$ C. de Graaf, I. de P. R. Moreira, F. Illas, and R. L. Martin, Phys. Rev. B 60, 3457 (1999).

${ }^{35}$ D. Muñoz, F. Illas, and I. de P. R. Moreira, Phys. Rev. Lett. 84, 1579 (2000).

${ }^{36}$ C. J. Calzado and J. P. Malrieu, Phys. Rev. B 63, 214520 (2001).

${ }^{37}$ C. J. Calzado, J. F. Sanz, J. P. Malrieu, and F. Illas, Chem. Phys. Lett. 307, 102 (1999).

${ }^{38}$ I. de P. R. Moreira, D. Muñoz, F. Illas, C. de Graaf, and M. A. Garcia-Bach, Chem. Phys. Lett. 345, 183 (2001).

${ }^{39}$ D. Muñoz, I. de P. R. Moreia, and F. Illas, Phys. Rev. B 65, 224521 (2002).

${ }^{40}$ C. Bloch, Nucl. Phys. 6, 329 (1958).

${ }^{41}$ J. des Cloizeaux, Nucl. Phys. 20, 321 (1960).

${ }^{42}$ Ph. Durand, Phys. Rev. A 28, 3184 (1983).

${ }^{43} \mathrm{Ph}$. de Loth, P. Cassoux, J. P. Daudey, and J. P. Malrieu, J. Am. Chem. Soc. 103, 4007 (1981).

${ }^{44}$ N. W. Winter and R. M. Pitzer, J. Chem. Phys. 89, 446 (1988).

${ }^{45}$ C. Sousa, F. Illas, and G. Pacchioni, J. Chem. Phys. 99, 6818 (1993).

${ }^{46}$ H. M. Evjen, Phys. Rev. 39, 675 (1932).

${ }^{47}$ P. Durand and J. C. Barthelat, Theor. Chim. Acta 38, 283 (1975).

${ }^{48}$ J. Miralles, J. P. Daudey, and R. Caballol, Chem. Phys. Lett. 198, 555 (1992).

${ }^{49}$ J. Miralles, O. Castell, R. Caballol, and J. P. Malrieu, Chem. Phys. 172, 33 (1993).

${ }^{50}$ J. M. Anglada, E. Besalú, and J. M. Bofill, Theor. Chem. Acc. 103, 163 (1999).

${ }^{51}$ J. M. Bofill, I. de P. R. Moreira, J. M. Anglada, and F. Illas, J. Comput. Chem. 21, 1375 (2000).

${ }^{52}$ J. M. Bofill and F. Illas, Mol. Phys. (to be published).

${ }^{53}$ F. Bernardi, M. Olivucci, J. J. McDouall, and M. A. Robb, J. Chem. Phys. 89, 6365 (1988).

${ }^{54}$ D. Maynau, M. A. Garcia-Bach, and J. P. Malrieu, J. Phys. (France) 47, 207 (1986).

${ }^{55}$ R. Ghailane, J. P. Malrieu, and D. Maynau, J. Phys. Chem. A 105, 3365 (2001). 